\title{
CS Research Square \\ Antimicrobial-specific response from resistance gene carriers in a diverse microbiome
}

\author{
Wisnu Adi Wicaksono \\ Peter Kusstatscher \\ Sabine Erschen \\ Tamara Reisenhofer-Graber \\ Martin Grube \\ Tomislav Cernava \\ Gabriele Berg
}

\section{Video Byte}

Keywords: Lichen microbiota, Peltigera polydactylon, Antimicrobial resistance, Metagenomic mining, Genome recovery, lichen, antibiotic resistance, antibiotics, ecosystem, Microbiome

Posted Date: February 25th, 2021

DOI: https://doi.org/10.21203/rs.3.rs-276091/v1

License: (c) (i) This work is licensed under a Creative Commons Attribution 4.0 International License.

Read Full License 


\section{Abstract}

Antimicrobial resistance (AMR) is an increasingly serious threat to global public health. New resistance mechanisms reduce our ability to treat common infectious diseases. Although microorganisms possessing AMR genes are thought to have emerged from natural habitats, better understanding is needed. A new study sought to examine the consequences of introducing antimicrobials into natural environments. Using lichen - a model for well-defined micro-ecosystems consisting of hundreds of microbial species - researchers evaluated changes in microbial communities following exposure to different antimicrobials. They found that the native lichen microbiome comprises highly diverse and lowabundance intrinsic antimicrobial resistance genes (ARGs) to cope with antimicrobial pressure. Antimicrobial-specific shifts occurred in the structure and function of the microbiome following 10 days of exposure to antimicrobials. Antimicrobial exposure resulted in dysbiosis due to a bloom in naturally low-abundance taxa with specific AMR features. Metagenomic analysis revealed that these species' intrinsic resistance mechanisms allow them to thrive when exposed to antimicrobials. While further research is needed to understand the response to antimicrobials in a wider variety of microbiomes, these results provide mechanistic insights into the community-level responses and dysbiosis that occur in native microbiota following antimicrobial exposure. 